\title{
On the elements of the orbit of 02387 .
}

After projecting the observations of $O \Sigma_{3} 8_{7}$ on millimetric paper I drew curves through the series of points representing the angles and the distances respectively, and improved them until the angles and distances confirmed each other, except for the period preceeding 1858, when it was impossible to bring them both into satisfactory agreement. The distances observed before 1858 seem to be too large judging by the angles, which define the orbit better in this instance. The following is the result. The distances deduced from the angles are placed in parenthesis.

\begin{tabular}{lrll|lll}
1845 & $120^{\circ} .2$ & 0.52 & $(0.41)$ & 1875 & $24^{\circ} .3$ & 0.43 \\
1850 & 105.6 & 0.54 & $(0.43)$ & 1880 & 13.4 & 0.47 \\
1855 & 92.2 & 0.49 & $(0.44)$ & 1885 & 3.1 & 0.52 \\
1860 & 79.8 & 0.44 & $(0.44)$ & 1890 & 354.3 & 0.56 \\
1865 & 65.5 & 0.41 & & 1895 & 346.7 & 0.62 \\
1870 & 47.8 & 0.41 & & & &
\end{tabular}

I now constructed four apparent ellipses as different inter se as possible. The resulting elements and ephemerides referred to 1900.0 were as follows:

\begin{tabular}{|c|c|c|c|c|c|c|c|c|}
\hline Orbit & \multicolumn{2}{|c|}{ I } & \multicolumn{2}{|c|}{ II } & \multicolumn{2}{|c|}{ III } & \multicolumn{2}{|c|}{ IV } \\
\hline$\delta$ & \multicolumn{2}{|c|}{$135^{\circ} 24^{\prime}$} & \multicolumn{2}{|c|}{$129^{\circ} 33^{\prime}$} & \multicolumn{2}{|c|}{ I $28^{\circ} 54^{\prime}$} & \multicolumn{2}{|c|}{$110^{\circ} 22^{\prime}$} \\
\hline$\gamma$ & & 6 & \multirow{2}{*}{\multicolumn{2}{|c|}{$\begin{array}{ll}65 & 45 \\
75 & 18\end{array}$}} & \multirow{2}{*}{\multicolumn{2}{|c|}{$\begin{array}{ll}59 & 10 \\
55 & 58\end{array}$}} & \multicolumn{2}{|c|}{$50 \quad 12$} \\
\hline$\lambda$ & \multirow{2}{*}{\multicolumn{2}{|c|}{$\begin{array}{l}77 \quad 3 \\
0.542\end{array}$}} & & & & & \multirow{2}{*}{\multicolumn{2}{|c|}{$\begin{array}{c}354 \quad 45 \\
0.393\end{array}$}} \\
\hline$e$ & & & \multicolumn{2}{|c|}{0.600} & \multicolumn{2}{|c|}{0.280} & & \\
\hline$P$ & \multirow{2}{*}{\multicolumn{2}{|c|}{$\begin{array}{c}75^{7} \cdot 79 \\
1839\end{array}$}} & \multirow{2}{*}{\multicolumn{2}{|c|}{$\begin{array}{l}90^{8} \\
183^{8}\end{array}$}} & \multicolumn{2}{|c|}{$134^{\frac{7}{2}}$} & \multirow{3}{*}{\multicolumn{2}{|c|}{$\begin{array}{c}260^{7} \\
1849.24 \\
0.89\end{array}$}} \\
\hline$T$ & & & & & 18 & & & \\
\hline$a$ & o": & & o." & & o."6 & & & \\
\hline 1845 & $130 \% 6$ & o" 34 & $121: 5$ & 0.42 & $115^{\circ}: 3$ & o. 55 & $117^{\circ} 1$ & ०."52 \\
\hline $185^{\circ}$ & I 10.0 & 0.40 & $107 \cdot 3$ & 0.46 & 105.2 & 0.53 & 105.4 & 0.52 \\
\hline 1855 & 92.7 & 0.42 & 93.7 & 0.45 & 93.8 & 0.49 & $93 \cdot 3$ & 0.50 \\
\hline 1860 & 75.9 & 0.42 & 78.8 & 0.43 & 80.4 & 0.45 & 79.9 & 0.47 \\
\hline I 865 & $59 \cdot 3$ & $0.4^{2}$ & $62 \cdot 3$ & $0.4 \mathrm{i}$ & 64.6 & 0.42 & 65.0 & 0.45 \\
\hline 1870 & $43 \cdot 3$ & 0.44 & 45.0 & $0.4 \mathrm{I}$ & 47.2 & $0.4 \mathrm{I}$ & 48.5 & 0.43 \\
\hline 1875 & 28.6 & 0.46 & 28.6 & 0.43 & 29.9 & 0.43 & 31.6 & 0.45 \\
\hline 1880 & 15.5 & 0.49 & 14.4 & 0.47 & 14.5 & 0.46 & I 5.8 & 0.47 \\
\hline I 885 & 3.9 & 0.52 & 2.6 & $0.5^{2}$ & I. 7 & $0.5 \mathrm{I}$ & 2.4 & $0.5 \mathrm{I}$ \\
\hline 1890 & 353.3 & 0.54 & 352.8 & 0.57 & 351.5 & 0.57 & $35 \times \cdot 3$ & 0.57 \\
\hline I 895 & $342 \cdot 3$ & 0.63 & 344.7 & 0.62 & $343 \cdot 1$ & 0.63 & $34^{2.4}$ & 0.63 \\
\hline
\end{tabular}

Orbits III and IV were constructed by aid of the distances not placed in parenthesis above. They represent the distances best. Orbits I and II, which were constructed by aid of the distances in parenthesis, represent the angles better than the distances. Orbits II and III are good, though II is decidedly the better. Orbits I and IV are bad, though $I$ is decidedly the worse. This shows the utility of publishing mere diagrams of a number of apparent orbits laid through the points representing the positions of the companion at various epochs. The orbits may be good or bad; that can- not be known till the observations (all the observations not merely an extract) have been compared with ephemerides computed from every one of the apparent orbits.

Nine years ago Prof. Glasenapp computed this orbit and his elements show some rough similarity to the orbits given above e.g. $P=1$ ro years, and also in the plane of the orbit $\left(\Omega=15^{1^{\circ}}, \gamma=44^{\circ}\right)$. The eccentricity determined by Glasenapp is rather small for the period, while the eccentricity given above is rather large.

\begin{tabular}{|c|c|c|c|c|c|c|c|}
\hline Observer & Epoch & $\theta_{0}$ & $\varrho_{o}$ & $\theta_{0}-\theta_{2}$ & $\varrho_{0}-\varrho_{2}$ & $\theta_{0}-\theta_{3}$ & $\varrho_{0}-\varrho_{3}$ \\
\hline O. Struve & 1842.70 & $124^{\circ} 4$ & 0.48 & $-6 \% 0$ & +0.12 & $+4: 3$ & -0.08 \\
\hline Mädler & 43.39 & I 18.3 & 0.53 & -9.3 & +0.15 & -0.3 & -0.03 \\
\hline O. Struve & 45.65 & I 30.0 & 0.49 & +10.3 & +0.06 & +15.7 & -0.06 \\
\hline Mädler & 47.73 & 119.4 & 0.60 & +5.8 & +0.15 & +9.3 & +0.06 \\
\hline O. Struve & 185 I. 63 & 104.4 & $0.4^{8}$ & +1.2 & +0.02 & +2.6 & -0.04 \\
\hline 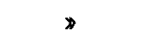 & 52.63 & 104.6 & 0.54 & +4.2 & +0.08 & +5.0 & +0.03 \\
\hline Dawes & 53.75 & 90.2 & $(0.53)$ & -7.3 & +0.07 & -6.9 & +0.03 \\
\hline Mädler & 53.78 & 94.7 & $0.5^{2}$ & -2.8 & +0.06 & -2.4 & +0.02 \\
\hline O. Struve & 54.69 & 95.8 & $0.5^{1}$ & +0.9 & +0.06 & +0.9 & +0.02 \\
\hline$»$ & 56.57 & 86.4 & $\left(0.5^{2}\right)$ & -3.3 & +0.08 & -3.9 & +0.04 \\
\hline Secchi & 56.83 & 91.9 & 0.30 & +3.0 & -0.14 & +2.4 & -0.18 \\
\hline O. Struve & 59.62 & 77.8 & $0.5^{1}$ & -2.2 & +0.08 & -3.7 & +0.06 \\
\hline
\end{tabular}




\begin{tabular}{|c|c|c|c|c|c|c|c|}
\hline Observer & Epoch & $\theta_{0}$ & $\varrho_{0}$ & $\theta_{o}-\theta_{2}$ & $\varrho_{0}-\varrho_{2}$ & $\theta_{o}-\theta_{3}$ & $\varrho_{0}-\varrho_{3}$ \\
\hline O. Struve & 1862.83 & $76: 9$ & $(0.57)$ & $+7 \circ 1$ & $+0 . " \mathrm{~s} 6$ & $+4: 9$ & +0.14 \\
\hline Dembowski & 65.53 & $5^{6.2}$ & - & -4.6 & - & -6.9 & - \\
\hline$»$ & 69.60 & $5^{2} \cdot 5$ & - & +5.8 & - & +3.7 & - \\
\hline$»$ & 1870.56 & 52.5 & - & +8.9 & - & +6.9 & - \\
\hline$»$ & 71.57 & 39.6 & - & -0.6 & - & -2.5 & - \\
\hline$»$ & 72.55 & 26.6 & - & -10.2 & - & -12.0 & - \\
\hline$»$ & 73.73 & 23.1 & - & -9.7 & - & -11.2 & - \\
\hline 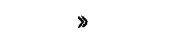 & 74.57 & 20.7 & 0.55 & -9.6 & +0.12 & -11.0 & +0.13 \\
\hline$\gg$ & $75.4 \mathrm{I}$ & 22.0 & 0.34 & -5.5 & -0.09 & -6.6 & -0.08 \\
\hline Schiaparelli & 75.57 & 23.2 & $0.3^{8}$ & -3.7 & -0.06 & -4.7 & -0.05 \\
\hline Hall & 76.76 & 20.8 & $0.4^{8}$ & -2.7 & +0.04 & -3.6 & +0.04 \\
\hline Dembowski & 77.59 & 16.2 & 0.49 & -5.0 & +0.04 & -5.7 & +0.05 \\
\hline Hall & 78.72 & 17.9 & 0.48 & +0.1 & +0.02 & -0.4 & +0.03 \\
\hline 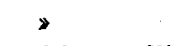 & 79.70 & 12.5 & $0.4^{8}$ & -2.8 & +0.01 & -3.0 & +0.02 \\
\hline Schiaparelli & 1880.68 & $11 \cdot 3$ & $0.4^{\circ}$ & -1.5 & -0.08 & -1.5 & -0.07 \\
\hline Hall & $8 \mathrm{I} .6 \mathrm{I}$ & 10.6 & 0.53 & +0.1 & +0.04 & +0.3 & +0.05 \\
\hline Hough & 82.69 & 8.5 & $0.4^{8}$ & +0.6 & -0.02 & +1.0 & -0.01 \\
\hline Hall & 82.70 & I 1.4 & 0.44 & +3.5 & -0.06 & +3.9 & -0.05 \\
\hline Engelmann & 83.13 & $5 \cdot 5$ & 0.40 & -1.3 & -0.10 & $-\mathbf{1} .0$ & -0.09 \\
\hline Hall & 83.63 & 8.9 & 0.54 & +3.3 & +0.03 & +3.9 & +0.04 \\
\hline Schiaparelli & 83.64 & 7.9 & $0.4^{8}$ & +2.3 & -0.03 & +2.9 & -0.02 \\
\hline Hall & 84.69 & 6.1 & 0.56 & +2.7 & +0.04 & +3.6 & +0.05 \\
\hline$\triangleright \quad$ & 85.74 & $3 \cdot 5$ & 0.53 & +2.4 & 0.00 & +3.3 & +0.01 \\
\hline Engelmann & 85.89 & $35^{8.3}$ & 0.62 & -2.5 & +0.09 & -1.5 & +0.10 \\
\hline Tarrant & 86.52 & 0.9 & 0.45 & +1.3 & -0.09 & +2.4 & -0.08 \\
\hline Perrotin & $86.5^{8}$ & 0.6 & 0.64 & +1.2 & +0.10 & +2.3 & +0.11 \\
\hline Hall & 86.62 & $4 \cdot 7$ & 0.54 & +5.3 & 0.00 & +6.4 & +0.01 \\
\hline H. Struve & 86.76 & 357.0 & $(0.50)$ & -2.1 & -0.04 & -1.0 & -0.04 \\
\hline Schiaparelli & 87.55 & 357.8 & 0.57 & +0.2 & +0.02 & +1.4 & +0.02 \\
\hline Hall & 88.73 & 356.3 & 0.54 & +1.0 & -0.02 & +2.3 & -0.02 \\
\hline H. Struve & 88.76 & 354.6 & 0.55 & -0.7 & -0.01 & +0.6 & -0.01 \\
\hline Schiaparelli & 8.8 .82 & 357.8 & $0.5^{2}$ & +2.6 & -0.04 & +3.9 & -0.04 \\
\hline Tarrant & 89.44 & 351.9 & $0.5^{8}$ & $-2 . I$ & +0.02 & -0.9 & +0.02 \\
\hline Hall & 89.66 & $35^{8.3}$ & 0.53 & +4.7 & -0.04 & +6.0 & -0.04 \\
\hline Schiaparelli & 89.87 & 353.7 & 0.54 & +0.5 & -0.03 & $+\quad 1.8$ & -0.03 \\
\hline Bigourdan & 1890.52 & 354.9 & - & +2.8 & - & +4.1 & - \\
\hline Hall & 90.62 & 355.9 & 0.58 & +4.0 & 0.00 & +5.3 & 0.00 \\
\hline Schiaparelli & 90.94 & 353.8 & 0.56 & +2.5 & -0.02 & +3.7 & -0.02 \\
\hline Bigourdan & $9 \times 62$ & 352.7 & 0.56 & +2.7 & -0.03 & +4.1 & -0.03 \\
\hline Tarrant & $9 \times 64$ & 349.9 & 0.54 & -0.1 & -0.05 & $+1 \cdot 3$ & -0.05 \\
\hline Comstock & 92.62 & 353.0 & 0.57 & +4.7 & -0.03 & +6.1 & -0.03 \\
\hline Bigourdan & 92.64 & $35^{2.1}$ & 0.65 & +3.8 & +0.05 & +5.2 & +0.05 \\
\hline Schiaparelli & 92.92 & 350.1 & 0.50 & +2.2 & -0.10 & +3.6 & -0.10 \\
\hline$»$ & 93.78 & 349.2 & 0.8 & +2.7 & +0.19 & +4.1 & +0.19 \\
\hline Comstock & 93.80 & 348.0 & 0.52 & +1.5 & -0.09 & +3.0 & -0.09 \\
\hline Bigourdan & 94.72 & $343 \cdot 3$ & 0.70 & $-\quad 1.8$ & +0.08 & -0.3 & +0.07 \\
\hline Schiaparelli & 94.77 & 349.0 & 0.6 & +3.9 & -0.02 & +5.4 & -0.03 \\
\hline Comstock & 95.72 & 346.7 & 0.55 & +3.1 & -0.07 & +4.7 & -0.08 \\
\hline Schiaparelli & 95.88 & 345.1 & $0.6 \mathrm{I}$ & +1.7 & -0.01 & +3.3 & -0.03 \\
\hline Comstock & 96.66 & 343.5 & 0.59 & +1.2 & -0.04 & +2.9 & -0.06 \\
\hline Hussey & 96.74 & 344.6 & $0.4^{6}$ & +2.4 & -0.17 & +4.1 & -0.19 \\
\hline Schiaparelli & 96.77 & 342.5 & 0.65 & +0.4 & +0.02 & +2.0 & 0.00 \\
\hline Doberck & $1897.7 \mathrm{I}$ & $342 \cdot 3$ & 0.76 & +1.5 & +0.12 & +3.2 & +0.10 \\
\hline
\end{tabular}

I submit that it has been proved that this system revolves in an orbit considerably inclined to the plane of vision in a period between 90 years and 150 years, and probably about 100 years.

Hongkong Observatory, 1898 July ro.

W. Doberck. 